\title{
Políticas de Admissão de Pacientes Oncológicos na UTI: Hora de Rever os Conceitos
}

\author{
Intensive Care Admission Policies for Critically \\ ill Cancer Patients: Time for a Reappraisal
}

A incidência de câncer vem aumentando na população brasileira e estima-se que neste ano haverá 472 mil novos casos no Brasil' ${ }^{1}$. Além disso, o câncer é a segunda causa mais comum de óbito na população brasileira, sendo superado apenas pelas doenças cardiovasculares ${ }^{1}$. Nos últimos anos, avanços nos cuidados dos pacientes com câncer possibilitaram maior probabilidade de controle ou cura da doença. Entretanto, o uso de tratamentos quimioterápicos e cirúrgicos mais agressivos implicam diretamente na maior utilização de leitos de UTI. Também, na última década, estudos têm demonstrado que os avanços recentes nos cuidados intensivos se traduziram na redução da mortalidade de pacientes críticos com câncer, mesmo em populações de maior risco como pacientes com sepse ou submetidos à ventilação mecânica ${ }^{2-5}$.

Entretanto, a internação na UTI de pacientes com câncer com complicações agudas graves ainda é motivo de controvérsia entre os intensivistas e os oncologistas uma vez que o seu prognóstico é freqüentemente considerado ruim a priori. Este conceito repetidamente gera recusa das internações dos pacientes nas UTI6. Uma avaliação ideal do mérito da internação de paciente com câncer na UTI necessita de complexa interação de fatores clínicos relacionados à complicação aguda, à neoplasia subjacente e às preferências e valores do paciente e seus familiares. Assim sendo, critérios atuais de admissão de pacientes oncológicos à UTI devem ser reavaliados.

Amendola e col. ${ }^{7}$, abordaram esta questão de modo interessante no subgrupo de pacientes cirúrgicos com câncer. Contudo, chama a atenção de que o grupo de pacientes não oncológicos possuírem mortalidade semelhante a dos pacientes oncológicos, mortalidade esta que esta pode ser considerada elevada em se tratando de intervenções cirúrgicas eletivas. Isto nos faz pensar na possibilidade de vieses de seleção que ocorrem sistematicamente nas UTI e que poderiam dificultar a comparação entre os grupos. Dentre estes possíveis vieses se destacam a possibilidade dos pacientes não oncológicos possuírem maior número e gravidade de comorbidades quando comparados aos oncológicos; e, em especial questões ligadas ao estadiamento da doença. Tais aspectos são de alta relevância uma vez que a presença de comorbidades graves encontra-se associada de modo independente com a letalidade em seis meses ${ }^{8}$ e a progressão do tumor parece ser um dos principais fatores de mau prognóstico em pacientes críticos com câncer ${ }^{4,9}$. Tais aspectos são de especial relevância ao considerarmos que há ainda um evidente preconceito à admissão de pacientes oncológicos, confirmado por estudos que demonstram que pacientes com prognósticos semelhantes (DPOC grave, insuficiência cardíaca classe IV-NYHA, cirróticos) possuem menor probabilidade de recusa de admissão na UTI e suporte terapêutico intensivo quando comparados a pacientes oncológicos de prognóstico semelhante ${ }^{10,11}$.

Outro aspecto a ser considerado é o das diferenças de subgrupos de pacientes oncológicos. Raras são as recusas para internar de pacientes em pós-operatório de intervenção cirúrgica eletiva. Os pacientes cirúrgicos, especialmente aqueles submetidos a cirurgias com intenção curativa, reconhecidamente têm melhor prognóstico quando comparados a pacientes clínicos ${ }^{4,8}$ e o benefício da internação na UTI é indiscutível. Por outro lado, a gravidade das disfunções orgânicas, o comprometimento da capacidade funcional (performance status) e o estado da neoplasia (estadiamento e fase da doença) são os principais determinantes do seu prognóstico e devem ser consideradas na discussão, propriedades da admissão a UTI.

Além disso, é fundamental expandir o conhecimento sobre o prognóstico dos pacientes com câncer que necessitam de cuidados intensivos. Nesse contexto, é preciso observar que os escores prognósticos não devem ser utilizados individualmente no processo de triagem nas UTI ${ }^{12}$. Em especial, o desempenho dos escores de prognóstico gerais e específicos em pacientes com câncer é ruim ${ }^{13,14}$. Entretanto, o conhecimento das características associadas com o prognóstico pode ajudar os profissionais de saúde em discussões sobre 
os pacientes e no seu aconselhamento e de seus familiares. Neste sentido a discussão atual entre experts na área tem evoluído no sentido de fornecer dados que forneçam informações sobre pacientes com melhor probabilidade de sobrevida, com qualidade de vida, após admissão a UTI. As recomendações que derivam da opinião de especialistas e que tem sido empregadas em centros de referência com UTI oncológicas sugerem sempre admitir a UTI pacientes em que haja dúvida acerca do prognóstico ${ }^{15}$. Nestes casos tratamentos com suporte pleno e agressivo por um tempo preestabelecido (em geral de aproximadamente cinco dias) em discussão com familiares, oncologistas e outros especialistas assistentes é empregado. Passado esse período o paciente é reavaliado e a evolução das alterações fisiológicas agudas e disfunções orgânicas presentes à admissão deverá nortear a decisão cobre o nível de suporte a ser mantido.

Somente com a solidificação de novos conhecimentos e atualização dos nossos conceitos sobre os pacientes críticos com câncer poderemos evitar que os cuidados intensivos deixem de ser oferecidos aos pacientes que deles possam beneficiar-se.

Jorge I. F. Salluh, MSc

Márcio Soares, PhD

Médico do CTI do Instituto Nacional de Câncer e do Hospital Barra D’or; Mestre em Clínica Médica/Pneumologia pela UFRJ.

Médico do CTI do Instituto Nacional de Câncer, Mestre e Doutor em Clínica Médica pela UFRJ. E-mail: jorgesalluh@yahoo.com.br

\section{REFERÊNCIAS}

01. Ministério da Saúde - Instituto Nacional de Câncer (INCA). Estimativas da Incidência e Mortalidade por Câncer no Brasil - 2003. Disponível em: $<$ www.inca.gov.br/estimativa/2006>

02. Larche J, Azoulay E, Fieux F et al - Improved survival of critically ill cancer patients with septic shock. Intensive Care Med, 2003;29:1688-1695.

03. Azoulay E, Alberti C, Bornstain C et al - Improved survival in cancer patients requiring mechanical ventilatory support: impact of noninvasive mechanical ventilatory support. Crit Care Med, 2001;29:519-525.

04. Soares M, Salluh JI, Spector N et al - Characteristics and outcomes of cancer patients requiring mechanical ventilatory support for $>24 \mathrm{~h}$. Crit Care Med, 2005;33:520-526.

05. Feres GA ; Salluh JIF ; Rocco JR ; Toscano, L. ; SOARES, M. . Características e Prognóstico de Pacientes com Câncer e Sepse Internados na Terapia Intensiva. In: Sepse 2006 - Da bancada a beira do leito, 2006 Rio de Janeiro. Anais do Congresso, 2006.

06. Thiery G, Azoulay E, Darmon M et al - Outcome of cancer patients considered for intensive care unit admission: a hospital-wide prospective study. J Clin Oncol, 2005;23:4406-4413.

07. Amendola CP, Almeida SLS, Horta VM et al - A doença oncológica não deve ser um fator limitante para admissão na uti de pacientes submetidos a cirurgias de alto risco. RBTI, 2006;18:268-275.

08. Soares M, Salluh JI, Ferreira CG et al - Impact of two different comorbidity on the 6-month mortality of critically ill cancer patients. Intensive Care Med, 2005;31:408-415.

09. Soares M, Salluh JI, Carvalho MS et al - Prognosis of critically ill patients with cancer and acute renal dysfunction. J Clin Oncol. 2006;20:40034010.

10. Lawrence VA, Clark GM - Cancer and resuscitation. Does the diagnosis affect the decision? Arch Intern Med, 1987;147:1637-1640.

11. Wachter RM, Luce JM, Hearst $\mathrm{N}$ et al - Decisions about resuscitation: inequities among patients with different diseases but similar prognoses. Ann Intern Med, 1989;111:525-532.

12. Lemeshow S, Klar J, Teres D - Outcome prediction for individual intensive care patients: useful, misused, or abused? Intensive Care Med, 1995;21:770-776.

13. Soares M, Fontes F, Dantas $\mathrm{J}$ et al - Performance of six severity-of-illness scores in cancer patients requiring admission to the intensive care unit: a prospective observational study. Crit Care, 2004;8:R194-R203.

14. Sculier JP, Paesmans M, Markiewicz E et al - Scoring systems in cancer patients admitted for an acute complication in a medical intensive care unit. Crit Care Med, 2000;28:2786-2792.

15. Azoulay E, Afessa B - The intensive care support of patients with malignancy: do everything that can be done. Intensive Care Med. 2006;32:3-5. 